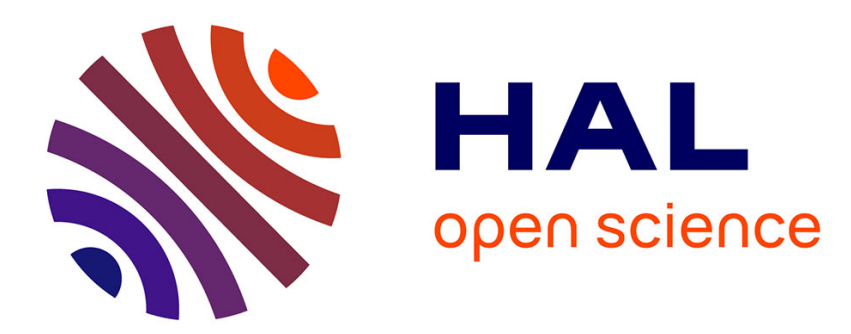

\title{
Bifurcation analysis of the thermocapillary convection in cylindrical liquid bridges
}

\author{
G Chen, A Lizée, B Roux
}

\section{To cite this version:}

G Chen, A Lizée, B Roux. Bifurcation analysis of the thermocapillary convection in cylindrical liquid bridges. Journal of Crystal Growth, 1997, 180 (3), pp.638-647. hal-01307132

\section{HAL Id: hal-01307132 \\ https://hal.science/hal-01307132}

Submitted on 28 Apr 2016

HAL is a multi-disciplinary open access archive for the deposit and dissemination of scientific research documents, whether they are published or not. The documents may come from teaching and research institutions in France or abroad, or from public or private research centers.
L'archive ouverte pluridisciplinaire HAL, est destinée au dépôt et à la diffusion de documents scientifiques de niveau recherche, publiés ou non, émanant des établissements d'enseignement et de recherche français ou étrangers, des laboratoires publics ou privés. 


\title{
Bifurcation Analysis of the Thermocapillary Convection in Cylindrical Liquid Bridges
}

\author{
G. Chen $^{1}$, A. LIzÉE ${ }^{2}$, ANd B. Roux ${ }^{1}$ \\ 1 Institut de Recherche sur les Phénomènes Hors Equilibre \\ UMR 6594 CNRS/Université d'Aix-Marseille I et II \\ Campus de Luminy-Case 903, 163 Avenue de Luminy \\ 13288 Marseille Cedex 9 France \\ 2 Center for Microgravity and Materials Research \\ University of Alabama in Huntsville \\ Huntsville, AL 35899, USA
}

Corresponding author address:

Dr. Gang Chen

Institut de Recherche sur les Phénomènes Hors Equilibre

UMR 6594 CNRS/Université d'Aix-Marseille I et II

Campus de Luminy-Case 903, 163 Avenue de Luminy

13288 Marseille Cedex 9 France

Phone: +33 (0)4 91411953

Fax: +33 (0)4 91419620

E-mail: chen@ajax.univ-mrs.fr

Journal of Crystal Growth 180, 638-647 (1997) 


\begin{abstract}
We consider the instability of the steady, axisymmetric thermocapillary convection in cylindrical liquid bridges. Finite-difference method is applied to compute the steady axisymmetric basic solutions, and to examine their linear instability to three-dimensional modal perturbations. The numerical results show that for liquid bridges of $O(1)$ aspect ratio $\Gamma$ (=length/radius) the first instability of the basic state is through either a regular bifurcation (stationary) or Hopf bifurcation (oscillatory), depending on the Prandtl number of the liquid. The bifurcation points and the corresponding eigenfunctions are predicted precisely by solving appropriate extended systems of equations. For very small Prandtl numbers, i.e. $\operatorname{Pr}<0.06$, the instability is of hydrodynamical origin that breaks the azimuthal symmetry of the basic state. The critical Reynolds number, for unit aspect ratio and insulated free surface, tends to be constant, $R e_{c} \rightarrow 1784$, as $\operatorname{Pr} \rightarrow 0$, the most dangerous mode being $m=2$. While for $\operatorname{Pr} \geq 0.1$, the instability takes the form of a pair of hydrothermal waves traveling azimuthally. The most dangerous mode is $m=3$ for $0.1 \leq \operatorname{Pr} \leq 0.8$ and $m=2$ for $\operatorname{Pr} \geq 0.9$. Dependence of the critical Reynolds number on other parameters is also presented. Our results confirm in large part the recent lineartheory results of Wanschura et al. [7] and provide a more complete stability diagram for the finite half-zone with a non-deformable free surface.
\end{abstract}

PACS numbers: 47.20.Dr, 47.27.Te, 02.60.Cb

Keywords: Instability, Thermocapillary convection, Liquid bridge, Critical surface-tension Reynolds number.

\title{
1 Introduction
}

Since the floating-zone crystal growth process is considered to be a promising method of obtaining high quality crystals in microgravity, the thermocapillary convection (driven by surface-tension gradients along the free-surface of the melt) in floating-zone melts has received much research interest during the last fifteen years. Experimental studies have been performed by many investigators in the so-called half-zone model, in which a cylindrical liquid bridge is suspended vertically between two, equal-diameter, differentially heated rods. High Prandtl number $(\operatorname{Pr}>1)$ fluids have been used in the experiments, although $\operatorname{Pr}$ of melt semiconductors and metals are about $10^{-2}$. It has been shown that if the temperature difference between two rods is sufficiently small, the flow is steady and axisymmetric. The flow pattern can be characterized by a single toroidal vortex 
with motion on the free surface whose direction goes from the hot cylinder toward the cold one. By increasing the temperature difference, this two-dimensional (2D) basic state undergoes a transition to time-dependent three-dimensional (3D) flow. The appropriate dimensionless parameter for fluids with large $\operatorname{Pr}$ is the Marangoni number. The azimuthal wavenumber $m$ of slightly supercritical flow was found to be primarily determined by the aspect ratio of liquid bridge $\Gamma$ (=height/radius). The instability of this convective mode has been believed to be responsible for the appearance of undesirable striations in the final crystals (see for example a review by Schwabe [1]).

Motivated by the experimental observations, several theoretical and numerical studies have been devoted to stability of the flow in a half-zone model, including energy stability theory $[2,3]$, linear stability analysis $[4,5,6,7]$ and 3D direct numerical simulation $[8,9]$. It is now well established (see Ref. [7]) that for liquid bridges of $O(1)$ aspect ratio the first instability is through either a regular bifurcation (stationary) or Hopf bifurcation (oscillatory), depending on the Prandtl number of the liquid. In both cases, the most dangerous mode is non-axisymmetric. This dependence of stability property on the Prandtl number has been observed for the first time by Rupp et al. [8] in their 3D simulation for $\Gamma=1.2$ and adiabatic free surface. In fact, they found that for small Prandtl numbers the time-dependent flow is preceded by a bifurcation to a steady $3 \mathrm{D}$ state, but no critical numbers for this bifurcation are given. For unit aspect ratio and a small Prandtl number $(\operatorname{Pr}=0.01)$, Levenstam and Amberg [9] found the critical Reynolds number for this bifurcation is 1960 with azimuthal wavenumber $m=2$.

In this paper, instead of conducting time-dependent simulation and directly computing the eigenvalues that characterize the regular or Hopf bifurcation, we used bifurcation theory combined with a finite difference method to determine the stability boundaries of the axisymmetric basic state in liquid bridges for a wide range of parameters. The stability of the basic state is examined by considering small three-dimensional disturbances in the normal form in the azimuthal direction. Resulting linearized disturbance equations form an eigenvalue problem for the complex growth rate. It depends on the parameters, azimuthal wavenumber $m$, Reynolds number $R e$, and basic state which in turn depends on parameters $R e, \operatorname{Pr}$ and $\Gamma$, and is solved numerically by successive Newton-Raphson iterations. The critical Reynolds number and the convective flow at the onset are predicted by solving an appropriate extended system of perturbation equations. Our stability calculations confirm in large part the recent linear-theory results of Wanschura et al. [7] and provide a more complete stability diagram for the finite half-zone with a non-deformable free surface. 


\section{Problem Formulation}

We consider a cylindrical liquid bridge of length $L$ held by surface tension forces between two parallel, coaxial solid rods of equal radii $R(>L / 2 \pi)$. A temperature difference $\Delta T$ is imposed over the liquid bridge by prescribing $T_{u}$ and $T_{l}$ at the upper and lower disks, respectively. The liquid is a Newtonian fluid with constant values of the viscosity $\mu$, reference density $\rho_{0}$, thermal diffusivity $\alpha$, and volume expansion coefficient $\beta ; \nu=\mu / \rho_{0}$ is the kinematic viscosity. The surface tension on the free surface is considered to be linearly depend on the temperature

$$
\sigma=\sigma_{0}-\gamma\left(T-T_{0}\right)
$$

where $\sigma_{0}$ is the mean surface tension at the reference temperature $T_{0}=\frac{1}{2}\left(T_{l}+T_{u}\right)$. For common liquids, we have $\gamma=-(d \sigma / d T)>0$, so that there is surface flow from the hot end toward the cold end. We assume that the free surface is not permitted to deform and has a perfectly cylindrical shape. This corresponds to requiring that the volume of the liquid zone is $\pi R^{2} L$ and that both capillary number, $C a=\gamma \Delta T / \sigma_{0}$, and Bond number, $B o=\rho_{0} g R^{2} / \sigma_{0}$, are asymptotically small, i.e., $C a \rightarrow 0$ and $B o \rightarrow 0$.

We consider the fully three-dimensional system in the usual cylindrical coordinate $(r, \phi, z)$ with origin in the center of the bottom end face. Gravity acts downward along the $z$-axis. The length $(r, z)$, velocity vector $\vec{u}=u \overrightarrow{e_{r}}+v \overrightarrow{e_{\phi}}+w \overrightarrow{e_{z}}$, pressure $p$, temperature difference $T-T_{0}$, and time $t$ are referred to scales $R, \gamma \Delta T / \mu, \gamma \Delta T / R, \Delta T=T_{u}-T_{l}$ and $R^{2} / \nu$, respectively. As a result, there arises the following dimensionless groups:

$$
R e=\frac{\gamma \Delta T R}{\mu \nu}, \operatorname{Pr}=\frac{\nu}{\alpha}, G r=\frac{g \beta \Delta T R^{3}}{\nu^{2}}, \Gamma=\frac{L}{R}, B i=\frac{h R}{\kappa} .
$$

These are respectively the surface-tension Reynolds number, Prandtl number, Grashof number, aspect ratio and surface Biot number in which $h$ is the heat transfer coefficient. Another useful dimensionless group is the Marangoni number, defined as $M a=\operatorname{RePr}$.

The governing equations for the flow and temperature fields in the liquid bridge are the Navier-Stokes, continuity and energy equations, subject to the Boussinesq approximation:

$$
\begin{gathered}
\frac{\partial \vec{u}}{\partial t}+\operatorname{Re} \vec{u} \cdot \nabla \vec{u}=-\nabla p+\nabla^{2} \vec{u}+\frac{G r}{R e} \theta \overrightarrow{e_{z}}, \\
\nabla \cdot \vec{u}=0 \\
\operatorname{Pr}\left(\frac{\partial \theta}{\partial t}+\operatorname{Re} \vec{u} \cdot \nabla \theta\right)=\nabla^{2} \theta .
\end{gathered}
$$


The boundary conditions applied to complete the problem specification are: at bottom and top solid surfaces, $z=0, \Gamma$,

$$
\begin{gathered}
\vec{u}=0, \\
\theta=\mp \frac{1}{2},
\end{gathered}
$$

on the free surface, $r=1$,

$$
\begin{gathered}
u=0, \\
\frac{\partial w}{\partial r}+\frac{\partial \theta}{\partial z}=0, \\
\frac{\partial v}{\partial r}-\frac{v}{r}+\frac{1}{r} \frac{\partial \theta}{\partial \phi}=0 \\
\frac{\partial \theta}{\partial r}+B i\left(\theta-\Theta_{a}(z)\right)=0
\end{gathered}
$$

Equation (2.7) is the kinematic condition. Equations (2.8) and (2.9) are the shear stress conditions. Heat transfer between liquid zone and surrounding gas is modelled by Equation (2.10) in which $\Theta_{a}(z)$ is the dimensionless ambient temperature. Positive values of $R e$ and $G r$ represent the system which is heated from above, while negative ones correspond to the system heated from below.

\section{Basic State Problem}

For sufficient weak axial temperature gradients, i.e. low surface-tension Reynolds numbers, equations (2.2-2.10) admit steady axisymmetric solutions with a flow in the $r$ - $z$-plane which can be characterized as a single toroidal vortex.

The steady axisymmetric basic state is denoted by $\vec{X}=\left\{\vec{U}(r, z)=U \overrightarrow{e_{r}}+W \overrightarrow{e_{z}}, P(r, z), \Theta(r, z)\right\}$, and satisfies the equations

$$
\begin{gathered}
\operatorname{Re}\left(U \frac{\partial U}{\partial r}+W \frac{\partial U}{\partial z}\right)+\frac{\partial P}{\partial r}-\left(\nabla^{2} U-\frac{U}{r^{2}}\right)=0 \\
R e\left(U \frac{\partial W}{\partial r}+W \frac{\partial W}{\partial z}\right)+\frac{\partial P}{\partial z}-\nabla^{2} W-\frac{G r}{R e} \Theta=0 \\
\frac{\partial U}{\partial r}+\frac{U}{r}+\frac{\partial W}{\partial z}=0 \\
\operatorname{Re} \operatorname{Pr}\left(U \frac{\partial \Theta}{\partial r}+W \frac{\partial \Theta}{\partial z}\right)-\nabla^{2} \Theta=0
\end{gathered}
$$

subject to the following boundary conditions:

$$
z=0, \Gamma: \quad U=W=0, \Theta=\mp \frac{1}{2},
$$




$$
\begin{gathered}
r=0: \quad U=\frac{\partial W}{\partial r}=\frac{\partial \Theta}{\partial r}=0 \\
r=1: \quad \\
U=0 \\
\frac{\partial W}{\partial r}+\frac{\partial \Theta}{\partial z}=0 \\
\frac{\partial \Theta}{\partial r}+\operatorname{Bi}\left(\Theta-\Theta_{a}(z)\right)=0 .
\end{gathered}
$$

The partial differential equations (3.1-3.4) are approximated by second-order central formulae for spatial derivatives and the boundary conditions (3.5-3.9) are approximated by second-order forward or backward difference approximations, on a non-uniform mesh. The resulting nonlinear algebraic system of equations, written in the vector form, reads

$$
\boldsymbol{f}(\vec{X} ; \lambda, \vec{\mu})=0
$$

where $\lambda$ is a specific parameter (Re in the present case) and $\vec{\mu}$ is the vector of the remaining parameters of the problem $(\operatorname{Pr}, G r, B i$ and $\Gamma)$. The nonlinear equations (3.10) are solved by successive Newton-Raphson iteration:

$$
\begin{gathered}
\boldsymbol{f}_{\vec{X}}\left(\vec{X}^{n} ; \lambda, \vec{\mu}\right) d \vec{X}^{n}=-\boldsymbol{f}\left(\vec{X}^{n} ; \lambda, \vec{\mu}\right) \\
\vec{X}^{n+1}=\vec{X}^{n}+d \vec{X}^{n}
\end{gathered}
$$

where $\boldsymbol{f}_{\vec{X}}=\partial \boldsymbol{f} / \partial \vec{X}$ is the Jacobian matrix. These iterations continue until the largest variation (relative) of any variable is less than some convergence tolerance which we set to $10^{-6}$. Depending on the parameters of the problem, 4 to 8 iterations can be required, provided that initial guess is close enough to the solution.

\section{Linear Stability Problem}

The stability of the basic state $\vec{X}$ is examined by considering small three-dimensional perturbations, denoted by $\overrightarrow{x^{\prime}}=\left\{\overrightarrow{u^{\prime}}(r, \phi, z, t), p^{\prime}(r, \phi, z), \theta^{\prime}(r, \phi, z)\right\}$, which satisfies the linearized perturbation equations

$$
\begin{gathered}
\frac{\partial \overrightarrow{u^{\prime}}}{\partial t}+\operatorname{Re}\left(\overrightarrow{u^{\prime}} \cdot \nabla \vec{U}+\vec{U} \cdot \nabla \overrightarrow{u^{\prime}}\right)+\nabla p^{\prime}-\nabla^{2} \overrightarrow{u^{\prime}}-\frac{G r}{R e} \theta^{\prime} \overrightarrow{e_{z}}=0, \\
\nabla \cdot \overrightarrow{u^{\prime}}=0, \\
\operatorname{Pr} \frac{\partial \theta^{\prime}}{\partial t}+\operatorname{Re} \operatorname{Pr}\left(\overrightarrow{u^{\prime}} \cdot \nabla \Theta+\vec{U} \cdot \nabla \theta^{\prime}\right)-\nabla^{2} \theta^{\prime}=0 .
\end{gathered}
$$


Since the basic state is axisymmetric, we can expand the perturbed velocity, pressure and temperature fields in the form

$$
\left[\begin{array}{c}
\overrightarrow{u^{\prime}} \\
p^{\prime} \\
\theta^{\prime}
\end{array}\right]=\sum_{m=-\infty}^{+\infty}\left[\begin{array}{l}
\vec{u}^{(m)}(r, z) \\
p^{(m)}(r, z) \\
\theta^{(m)}(r, z)
\end{array}\right] e^{\sigma^{(m)} t+j m \phi},
$$

where $j=\sqrt{-1}, m$ is the (integer) azimuthal wave number, and $\sigma^{(m)}$ is the complex growth rate of the corresponding perturbation mode. Substituting (4.4) into (4.1-4.3) yields, for each value of $m$, a set of equations having the similar form to (4.1-4.3) except that $\partial / \partial t \rightarrow \sigma^{(m)}$ and $\partial / \partial \phi \rightarrow j m$. The resulting discrete form of the equations, obtained by using finite-difference approximations for derivatives of the field variables, can be written as a generalized matrix eigenvalue problem

$$
\boldsymbol{g}(\vec{x}, \vec{X}, R e, m, \vec{\mu}) \equiv A \vec{x}=\sigma B \vec{x},
$$

where $\vec{x} \equiv(u, j v, w, p, \theta)^{T}$ denotes a vector consisting of disturbance velocity, pressure and temperature. $A \equiv \boldsymbol{g}_{\vec{x}}(\vec{X}, R e, m, \vec{\mu})$ is a real-valued, non-symmetric matrix, and $B$ is a (singular) real-valued, diagonal matrix. The Jacobian matrix $\boldsymbol{g}_{\vec{x}}$ is not the same as for the stationary solution $\boldsymbol{f}_{\vec{X}}$ except for the case of $m=0$, i.e. two-dimensional perturbations. In such a case, we make no difference between the two Jacobian matrices and either of them can be used in the analysis.

The complex growth rate $\sigma\left(=\sigma_{r}+j \omega\right.$, where $\omega$ is the angular frequency of the oscillations) depends on the parameters $m$ and $R e$, and the axisymmetric basic state which in turn depends on the parameters $R e$, and $\vec{\mu}(\operatorname{Pr}, G r, B i$ and $\Gamma)$. If $\vec{\mu}$ is fixed, then $\sigma=\sigma(R e, m)$. The condition that the 'leading' eigenvalue (i.e. the eigenvalue with the maximum real part) has $\sigma_{r}=0$ defines a neutral curve $\operatorname{Re}(m)$ ( $m$ should be an integer number). The minimum of $R e(m)$ over all $m$ determines a critical value of $R e$, say $R e_{c}$, and corresponding values of $m$ and $\omega$, say $m$ and $\omega_{c}$. The basic state is stable to such small disturbances if $R e<R e_{c}$, but for $R e>R e_{c}$ there exists values of $m$ such that there is at least one eigenvalue $\sigma$ with $\sigma_{r}>0$, so that the corresponding eigenmode will grow and is linearly unstable. If the corresponding imaginary part of this leading eigenvalue is zero, i.e. $\omega=0$, at $R e_{c}$, then we have a regular bifurcation to a new branch of steady solutions. However, if it is non-zero, so that a leading complex conjugate pair of eigenvalues crosses the imaginary axis into the right-half plane, then we have a Hopf bifurcation to a time-periodic solution from the stationary solution branch, whose frequency at the onset of this instability is determined by the magnitude of the imaginary part of the eigenvalue $\omega_{c}$. In either case, the spatial form of the bifurcating solution is determined by the eigenspace of the crossing eigenvalue or conjugate pair of eigenvalues. 


\section{$5 \quad$ Extended Systems for Locating Bifurcation Points}

We describe below two appropriate extended systems to locate, respectively, regular (stationary) bifurcations and Hopf (oscillatory) bifurcations. The resulting nonlinear algebraic set of equations are solved by Newton's method to give both the solution at the bifurcation point and the value of the bifurcation parameter.

\subsection{Stationary bifurcation points}

The system of equations we use to calculate a stationary bifurcation point is the one proposed by Moore and Spance [10] for locating limit points:

$$
\begin{gathered}
\boldsymbol{f}(\vec{X}, \lambda, \vec{\mu})=0, \\
\boldsymbol{g}(\vec{x}, \vec{X}, \lambda, m, \vec{\mu})=0, \\
\left(\vec{e}_{k}\right)^{T} \cdot \vec{x}=1,
\end{gathered}
$$

where the last equation defines a normalization condition of the eigenvector $\vec{x}$ (note that $\vec{x} \in \vec{R}), \vec{e}_{k}$ is the unit vector with components $\left(\vec{e}_{k}\right)_{i}=\delta_{i k}$. We solve the above system for the basic state $\vec{X}$, the bifurcating eigenvector $\vec{x}$ and the critical value of one specific parameter $\lambda$ (Re in the present case), at the prescribed values of all the other parameters $m$ and $\vec{\mu}$.

\section{$5.2 \quad$ Hopf bifurcation points}

The determination of the bifurcation point for a Hopf bifurcation is more complicated than for a stationary one. At a Hopf bifurcation point a complex-conjugate pair of eigenvalues with non-zero imaginary parts, $\pm j \omega$, crosses the imaginary axis. That bifurcation is the solution of the following extended system [11] of equations:

$$
\begin{gathered}
\boldsymbol{f}(\vec{X}, \lambda, \vec{\mu})=0, \\
\boldsymbol{g}(\vec{x}, \vec{X}, \lambda, m, \vec{\mu})=j \omega B \vec{x}, \\
\left(\vec{e}_{k}+j \vec{e}_{k}\right)^{T} \cdot \vec{x}=j,
\end{gathered}
$$

where the last equation in the set is a normalization condition for the eigenvector $\vec{x}$ (note that $\vec{x} \in \vec{C}$ ). We solve for the basic state $\vec{X}$, the bifurcating eigenvector $\vec{x}$, the angular frequency $\omega$ and the critical value of the bifurcation parameter $\lambda$, i.e. $R e$, at the fixed values of all the other parameters $m$ and $\vec{\mu}$. 


\section{Results and Discussion}

The principal results of the calculations for $\Gamma=1, B i=0$ and $G r=0$ are presented in

Table 1. As can be seen, the Hopf frequency becomes non zero when $\operatorname{Pr} \geq 0.1$; indicating that there exists two distinctly different instabilities of the base state, namely, stationary instability $\left(\omega_{c}=0\right.$, for $\left.\operatorname{Pr}<0.1\right)$ and oscillatory instability $\left(\omega_{c} \neq 0\right.$, for $\left.\operatorname{Pr} \geq 0.1\right)$.

\subsection{Stationary instability}

For small Prandtl numbers $(\operatorname{Pr}<0.06)$, the basic state losses its stability to a stationary non-axisymmetric disturbance and thus bifurcates into a three-dimensional steady state. The most dangerous mode has azimuthal wavenumber $m=2$. When $\operatorname{Pr}$ goes to zero, the critical Reynolds number remains finite and tends to be a constant $R e_{c}=1784$; indicating that the instability is hydrodynamic in origin. This limiting critical Reynolds number deviates less than $0.5 \%$ from that of Wanschura et al. [7] $\left(R e_{c}=1793\right)$.

Typical critical disturbance temperature and flow fields at $\operatorname{Pr}=0.02$ are presented in figure 1. It is seen from fig.1(a) that in a horizontal cut at $z=0.5$ the disturbance temperature field consists of two hot spots at $\phi=\pi / 2$ and $3 \pi / 2$, and two cold spots at $\phi=0$ and $\pi$. Fig.1(b) shows the critical disturbance flow field on the free surface. It is of interest to note that the azimuthal flow on the free surface is from the cold spots towards the hot spots. This is opposite to what would be expected from the thermocapillary effect which would drive an azimuthal flow away from hot spots and towards cold spots. Thus for small Prandtl numbers, the thermocapillary effect acts as a weak force counteracting the azimuthal flow.

The energy analysis by Wanschura et al. [7] shows that the small $\mathrm{Pr}$ instability mechanism is due to an energy feed by a disturbance flow normal to the basic axial shear flow. The presence of rigid rods is responsible for such a mechanism. In fact, $\mathrm{Xu}$ and Davis [4] did not find this type of instability in an infinitely long cylindrical liquid bridge $(\Gamma \rightarrow \infty)$ when $\operatorname{Pr} \rightarrow 0$.

The effect of the aspect ratio on the critical Reynolds number is shown in figure 2 for $\operatorname{Pr}=0.02, B i=0$ and $G r=0$. It can be seen that the presence of rigid walls stabilizes the basic flow and thus the instability is suppressed as the aspect ratio $\Gamma$ increases. Plotted is also a fit function giving the dependence of the critical Reynolds number on the aspect ration. For this Prandtl number, we obtained the following dependence by using a linear 
regression:

$$
R e_{c}=2160 \Gamma^{-1.26}
$$

As the aspect ratio increases from $\Gamma=0.4$ to 1.8 , the critical azimuthal wavenumber decreases from $m=4$ to $m=1$. In an attempt to give a relation between aspect ratio and wavenumber, we multiple $\Gamma$ by the corresponding critical azimuthal mode $m$ and obtain:

$$
1.6 \leq m \Gamma \leq 3.2
$$

Note that Preisser et al. [12] found the product of $m \Gamma=2.2$ in their experimental study with a fluid of $\operatorname{Pr}=7$. This value is in the range given by equation (6.2). However, it must be pointed out that the instability for $\operatorname{Pr}=7$ was not a stationary but an oscillatory one as shown below.

\subsection{Oscillatory instability}

As shown in Table 1, when $\operatorname{Pr} \geq 0.1$, another type of instability appears. The instability is oscillatory with Hopf frequency $\omega_{c}$. For $\Gamma=1, B i=0$ and $G r=0$, we found two different critical modes with azimuthal wavenumber $m=2$ for $\operatorname{Pr} \geq 0.9$ and $m=3$ for $0.1 \leq \operatorname{Pr}<0.9$. The Hopf frequency $\omega_{c}$ has the same dependence on Prandtl number as critical Reynolds number, i.e., it decreases with increasing Prandtl number. Our result of $\operatorname{Re}_{c}(\operatorname{Pr}=1)=2532$ deviates less than $2 \%$ from that of Neitzel et al. [5] $\left(R e_{c}=\right.$ 2484 ) and agrees within $2 \%$ with the result given by Wanschura et al. [7] $\left(R e_{c}=2539\right)$. However, the latter authors obtained much larger critical Reynolds numbers in the range of $0.5 \leq \operatorname{Pr} \leq 0.7$, while at $\operatorname{Pr}=0.8$ they found a much lower critical Reynolds number $\left(R e_{c}=4086\right)$. This can be explained by the fact that these authors obtained a different critical azimuthal wavenumber, namely, $m=2$.

The dependence of the critical Marangoni number $M a_{c}=\operatorname{Re} e_{c} \operatorname{Pr}$ on $\operatorname{Pr}$ is shown in figure 3. It is noted that $M a_{c}$ is nearly a linear function with $\operatorname{Pr}$ for $\operatorname{Pr}>1$ and it increases with increasing Prandtl number. This indicates that the Marangoni number is a more appropriate dimensionless parameter to characterize the oscillatory instability in the range of large Prandtl number $(\operatorname{Pr}>1)$. In the range $0.1 \leq \operatorname{Pr}<1$, the $M a_{c}$ curve exhibits a dramatical change with a peak at $\operatorname{Pr}=0.7$. We note that Rupp et al. [8] also observed an order of magnitude change of $M a_{c}$ in this region of the stability diagram. This

phenomenon may be due to the fact that there is a jump of instability (from stationary to oscillatory) near $\operatorname{Pr}=0.1$ and the critical mode has an azimuthal wavenumber $m=3$ while it becomes $m=2$ for $\operatorname{Pr} \geq 0.9$. 
A representative example for the critical disturbance flow and temperature field at $\operatorname{Pr}=7$ is given in figures 4(a) and 4(b). In a horizontal cut at $z=0.5$ the disturbance flow field consists of four cells, one in each quadrant, but slightly shifted in the positive $\phi$-direction. The surface flow originates from the hot surface spots at $\phi=0$ and $\pi$, and moves toward the cold surface spots. It can be seen that there exists a phase shift between the disturbance flow and temperature field. Such a phase shift can only be maintained if the whole disturbance propagates azimuthally. This is confirmed in figure 5 in which we see that the disturbance temperature field on the free surface propagates in the positive $\phi$-direction (from left to right in the figure). Moreover, we note that during the disturbance propagation two hot spots and two cold spots remain at the same horizontal position. This indicates that the large Prandtl number instability is due to the surface hydrothermal wave traveling azimuthally. Due to the presence of endwalls, we did not find an axial component of this hydrothermal wave as observed by $\mathrm{Xu}$ and Davis [4] in an infinitely long cylindrical liquid bridge.

The heat transfer from the liquid bridge to its surrounding is modelled by equation (2.10), where the ambient temperature $\Theta_{a}(z)$ is assumed to be the conducting profile. The effect of cooling on the stability of the basic state may be observed by examining the data in Table 2 for $\operatorname{Pr}=1, \Gamma=1, G r=0$ and varying Biot number $B i$. It is clear that increasing heat loss at the free surface stabilizes the flow, in agreement with the linear theory analysis in [4]. As the Biot number increases, the Hopf frequency of the hydrothermal wave increases and modes with higher azimuthal wavenumber $m$ become critical.

The effect of weak buoyancy forces on the stability limit can be seen from data in Table 3 for $\operatorname{Pr}=1, B i=0, \Gamma=1$ and varying values of Grashof number. These positive values of the Grashof number correspond to heating from above in our notation. It is seen that critical Reynolds number decreases with increasing Grashof number, indicating the destabilizing effect of buoyancy force. This trend is in contrast to the speculation of $\mathrm{Xu}$ and Davis [4], who reasoned that the axial temperature gradient in the half-zone experiments (heated from above) would have a stabilizing effect. But our result is in agreement with the calculations by Neitzel et al. [5].

\section{Conclusion}

We have investigated numerically the stability of the steady, axisymmetric thermocapillary convection in non-deformable cylindrical liquid bridges. The numerical results show that 
for liquid bridges of $O(1)$ aspect ratio the first instability of the basic state is through either a regular bifurcation (stationary) or Hopf bifurcation (oscillatory), depending on the Prandtl number of the liquid. The bifurcation points and the corresponding eigenfunctions have been determined by solving appropriate extended systems of equations. For very

small Prandtl numbers, i.e., $\operatorname{Pr}<0.06$, the basic state loses stability to a stationary disturbance with azimuthal wavenumber 2. It has been shown that the instability is of hydrodynamical nature. While for $\operatorname{Pr} \geq 0.1$, the instability is oscillatory which takes the form of a pair of hydrothermal waves traveling azimuthally. Two different critical azimuthal wavenumbers have been found, depending on the Prandtl number. The most dangerous mode is $m=3$ for $0.1 \leq \operatorname{Pr} \leq 0.8$ and $m=2$ for $\operatorname{Pr} \geq 0.9$.

For large Prandtl numbers, we found that heat loss at the free surface leads to a stabilization of the base state. But buoyancy force has a destabilizating effect, this is may be due to the strong radial temperature gradients of the basic state.

For small Prandtl numbers, we found a strong coupling of the azimuthal wavenumber of the instability with the aspect ratio of liquid bridge. The product of the most dangerous mode with the aspect ratio, $m \Gamma$, has been found to be in the range of 1.6 and 3.2, indicating that the most unstable mode has some feature of pattern selection.

Our results confirm in large part the recent linear-theory results of Wanschura et al. [7] and provide a more complete stability diagram for the finite half-zone with a non-deformable free surface.

\section{Acknowledgments}

This work has been supported by the Centre National d'Etudes Spatiales (CNES) which is gratefully acknowledged. 


\section{References}

[1] D. Schwabe, Crystals (Springer-Verlag, Berlin, 1988), Vol. 11, p. 75.

[2] Y. Shen, G.P. Neitzel, D.F. Jankowski and H.D. Mittelmann, J. Fluid Mech. 217 (1990) 639.

[3] G.P. Neitzel, C.C Law, D.F. Jankowski and H.D. Mittelmann, Phys. Fluids A 3 (1991) 2841.

[4] J.J. Xu and S.H. Davis, Phys. Fluids 27 (1984) 1102.

[5] G.P. Neitzel, K.T. Chang, D.F. Jankowski and H.D. Mittelmann, Phys. Fluids A 5 (1993) 108.

[6] H.C. Kuhlmann and H.J. Rath, J. Fluid Mech. 247 (1993) 247.

[7] M. Wanschura, V.M Shevtsova, H.C. Kuhlmann and H.J. Rath, Phys. Fluids 7 (1995) 912.

[8] R. Rupp, G. Müller and G. Neumann, J. Crystal Growth 97 (1989) 34.

[9] M. Levenstam and G. Amberg, J. Fluid Mech. 297 (1995) 357.

[10] G. Moore and A. Spence, SIAM J. Numer. Anal. 17 (1980) 567.

[11] A. Griewank and G. Reddien, IMA J. Numer. Anal. 3 (1983) 295.

[12] F. Preisser, D. Schwabe and A. Scharmann, J. Fluid Mech. 126 (1983) 545. 
TABLE 1

Critical Reynolds number $R e_{c}$, Hopf frequency $\omega_{c}$, and corresponding azimuthal mode $m$ as a function of Prandtl number $\operatorname{Pr}$ for $\Gamma=1, B i=0$ and $G r=0$.

\begin{tabular}{crrr|rrrr}
\hline $\operatorname{Pr}$ & $R e_{c}$ & $\omega_{c}$ & $m$ & $\operatorname{Pr}$ & $R e_{c}$ & $\omega_{c}$ & $m$ \\
\hline $10^{-10}$ & 1784 & 0 & 2 & 0.4 & 9528 & 277.4 & 3 \\
$10^{-6}$ & 1784 & 0 & 2 & 0.5 & 9033 & 250.2 & 3 \\
$10^{-3}$ & 1793 & 0 & 2 & 0.6 & 8543 & 222.4 & 3 \\
0.01 & 1892 & 0 & 2 & 0.7 & 7566 & 187.1 & 3 \\
0.02 & 2054 & 0 & 2 & 0.8 & 4769 & 120.7 & 3 \\
0.03 & 2299 & 0 & 2 & 0.9 & 3040 & 73.8 & 2 \\
0.04 & 2704 & 0 & 2 & 1.0 & 2532 & 64.7 & 2 \\
0.05 & 3528 & 0 & 2 & 2.0 & 1398 & 40.1 & 2 \\
0.055 & 4541 & 0 & 2 & 3.0 & 1127 & 32.4 & 2 \\
0.056 & 4939 & 0 & 2 & 4.0 & 995 & 28.3 & 2 \\
0.057 & 5590 & 0 & 2 & 5.0 & 923 & 25.7 & 2 \\
0.1 & 16201 & 450.0 & 3 & 6.0 & 892 & 24.1 & 2 \\
0.2 & 13278 & 391.4 & 3 & 7.0 & 869 & 22.7 & 2 \\
0.3 & 10524 & 315.9 & 3 & 8.0 & 855 & 21.9 & 2 \\
\hline
\end{tabular}

TABLE 2

Critical Reynolds number $R e_{c}$, Hopf frequency $\omega_{c}$, and corresponding azimuthal mode $m$ as a function of Biot number $B i$ for $\operatorname{Pr}=1, \Gamma=1$, and $G r=0$.

\begin{tabular}{crrc}
\hline$B i$ & $R e_{c}$ & $\omega_{c}$ & $m$ \\
\hline 0 & 2523 & 74.7 & 2 \\
0.1 & 2624 & 66.4 & 2 \\
0.5 & 3183 & 75.2 & 2 \\
1 & 4618 & 115.2 & 3 \\
2 & 7613 & 182.0 & 3 \\
5 & 10700 & 260.0 & 3 \\
\hline
\end{tabular}


TABLE 3

Critical Reynolds number $R e_{c}$ and Hopf frequency $\omega_{c}$, as a function of Grashof number $G r$ for $\operatorname{Pr}=1, \Gamma=1$, and $B i=0$. The azimuthal wavenumber is $m=2$.

\begin{tabular}{ccc}
\hline$G r$ & $R e_{c}$ & $\omega_{c}$ \\
\hline 0 & 2523 & 64.7 \\
500 & 2500 & 63.2 \\
$10^{3}$ & 2474 & 62.1 \\
$2 \times 10^{3}$ & 2467 & 60.5 \\
\hline
\end{tabular}




\section{List of Figures}

1 (a) Critical disturbance temperature field in a horizontal cut at $z=0.5$ (hot spot maxima are indicated by "+" and cold spot minima by "-") and (b) critical disturbance flow field on the free surface for $R e_{c}=2054$, $\operatorname{Pr}=0.02, \Gamma=1, B i=0$ and $G r=0 \ldots \ldots \ldots$. . . . . . . 17

2 Dependence of the critical Reynolds number $R e_{c}$ and corresponding azimuthal wavenumber $m$ on the aspect ratio $\Gamma$ for $\operatorname{Pr}=0.02, B i=0$ and $G r=0$. Numerical results are shown by "o". Plotted solid line is a fit function given by $R e_{c}=2160 \Gamma^{-1.26}$. . . . . . . . . . . . . . . . . 18

3 Dependence of the critical Marangoni number $M a_{c}$ on the Prandtl number $\operatorname{Pr}$ for $\Gamma=1, B i=0$ and $G r=0 \ldots \ldots \ldots$

4 Critical disturbance flow (a) and temperature field (b) in a horizontal cut at $z=0.5$ for $R e_{c}=869, \omega_{c}=22.7, \operatorname{Pr}=7, \Gamma=1, B i=0$ and $G r=0 . \quad .20$

5 Time-dependence of the critical disturbance temperature field on the free surface (surface hydrothermal wave) for $R e_{c}=869, \omega_{c}=22.7, \operatorname{Pr}=7$, $\Gamma=1, B i=0$ and $G r=0$. The isotherms are shown at times 0 (a), $1 / 4$ (b), $1 / 2(\mathrm{c})$ and $3 / 4(\mathrm{~d})$, in units of $2 \pi / \omega_{c} \ldots \ldots \ldots . \ldots 22$ 

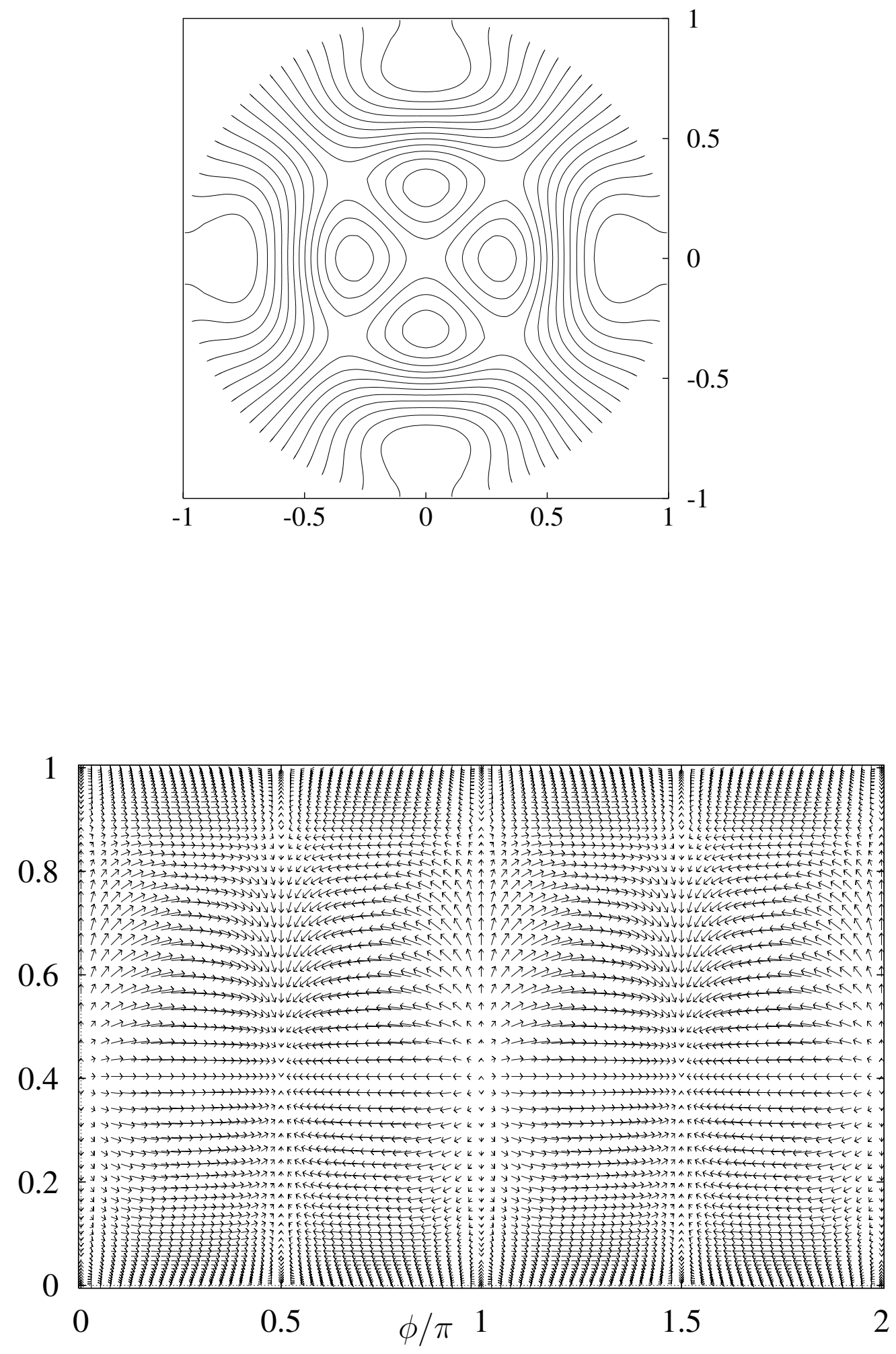

Figure 1: (a) Critical disturbance temperature field in a horizontal cut at $z=0.5$ (hot spot maxima are indicated by "+" and cold spot minima by "-") and (b) critical disturbance flow field on the free surface for $R e_{c}=2054, \operatorname{Pr}=0.02, \Gamma=1, B i=0$ and $G r=0$. 


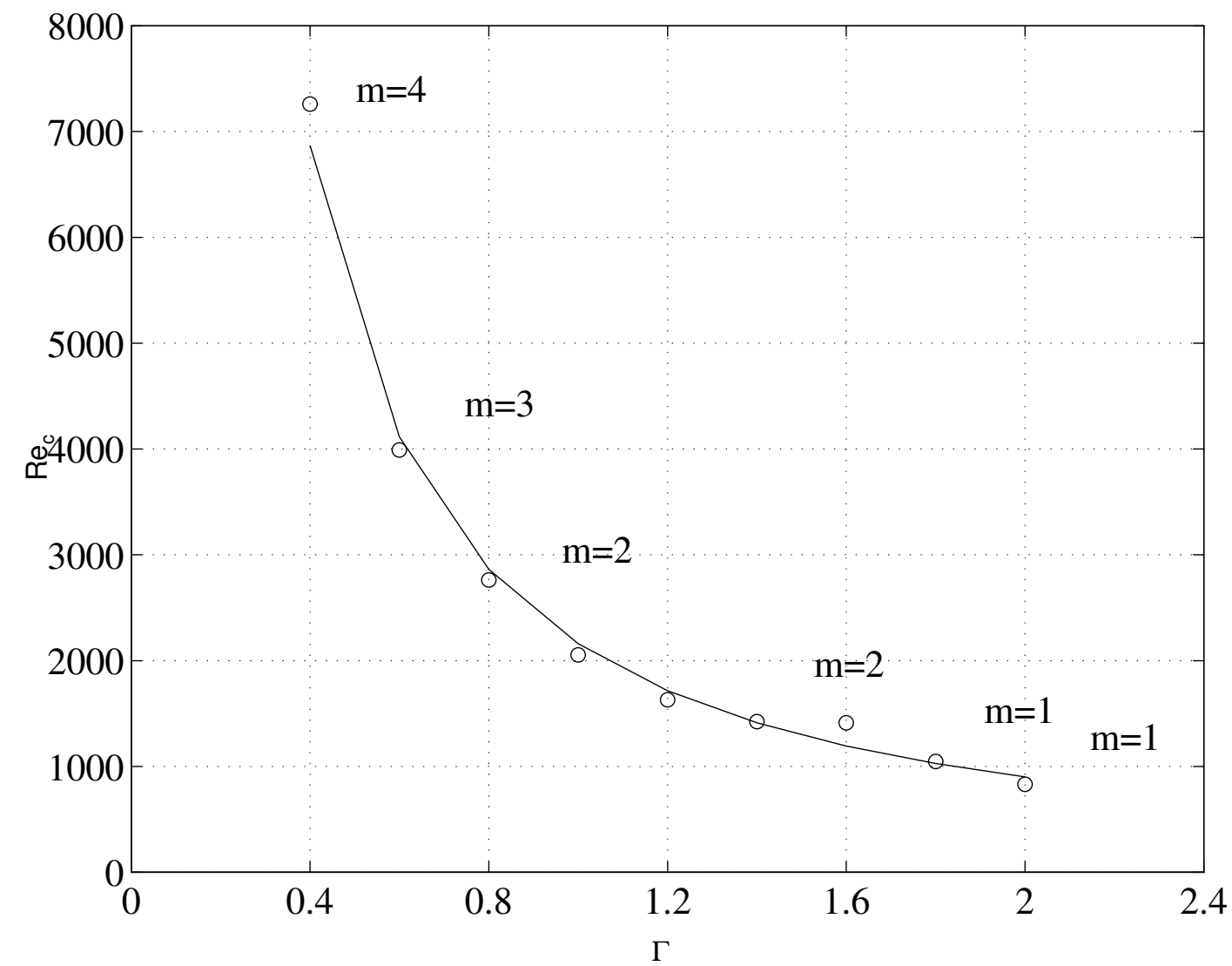

Figure 2: Dependence of the critical Reynolds number $R e_{c}$ and corresponding azimuthal wavenumber $m$ on the aspect ratio $\Gamma$ for $P r=0.02, B i=0$ and $G r=0$. Numerical results are shown by "o". Plotted solid line is a fit function given by $R e_{c}=2160 \Gamma^{-1.26}$. 


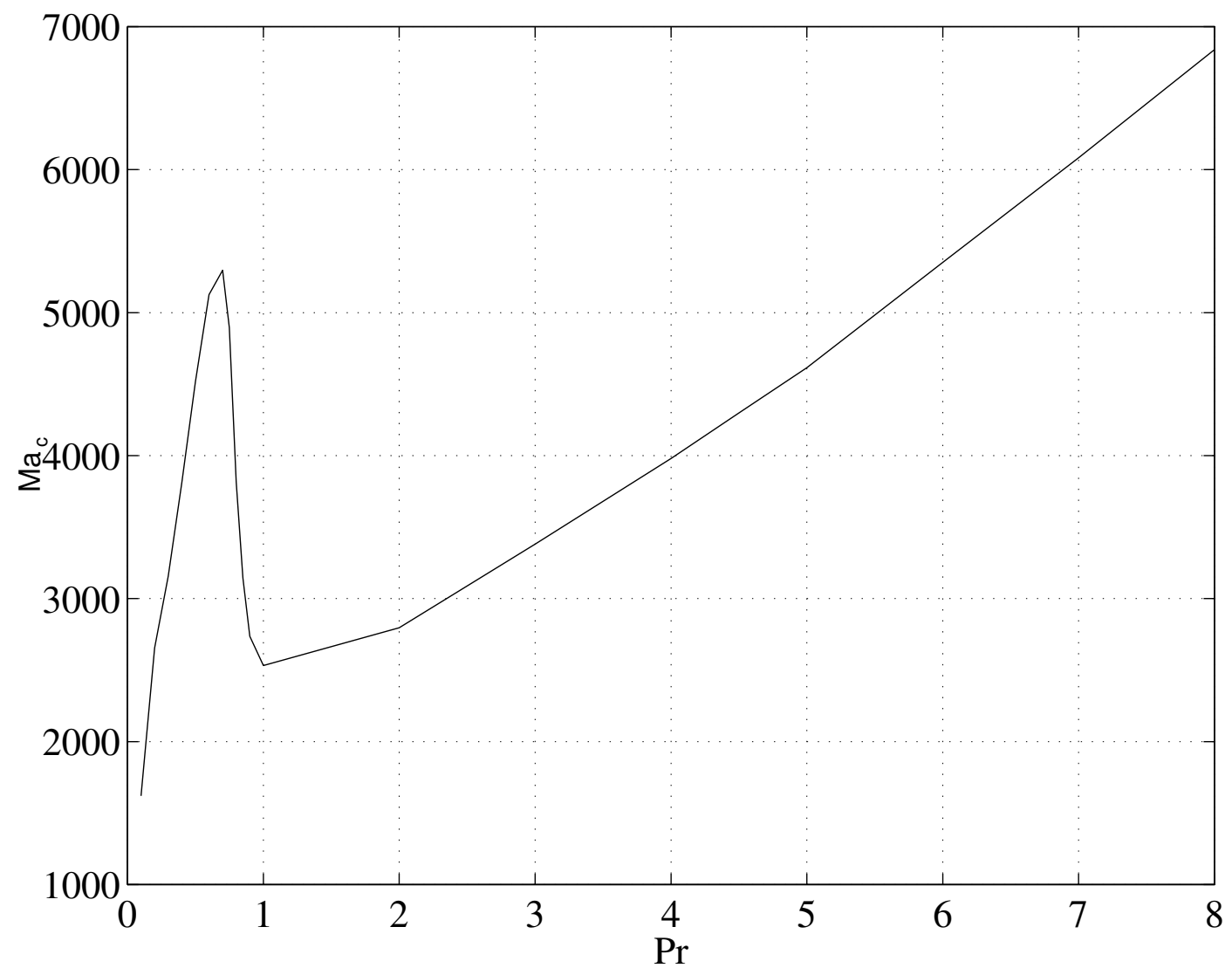

Figure 3: Dependence of the critical Marangoni number $M a_{c}$ on the Prandtl number $\mathrm{Pr}$ for $\Gamma=1, B i=0$ and $G r=0$. 

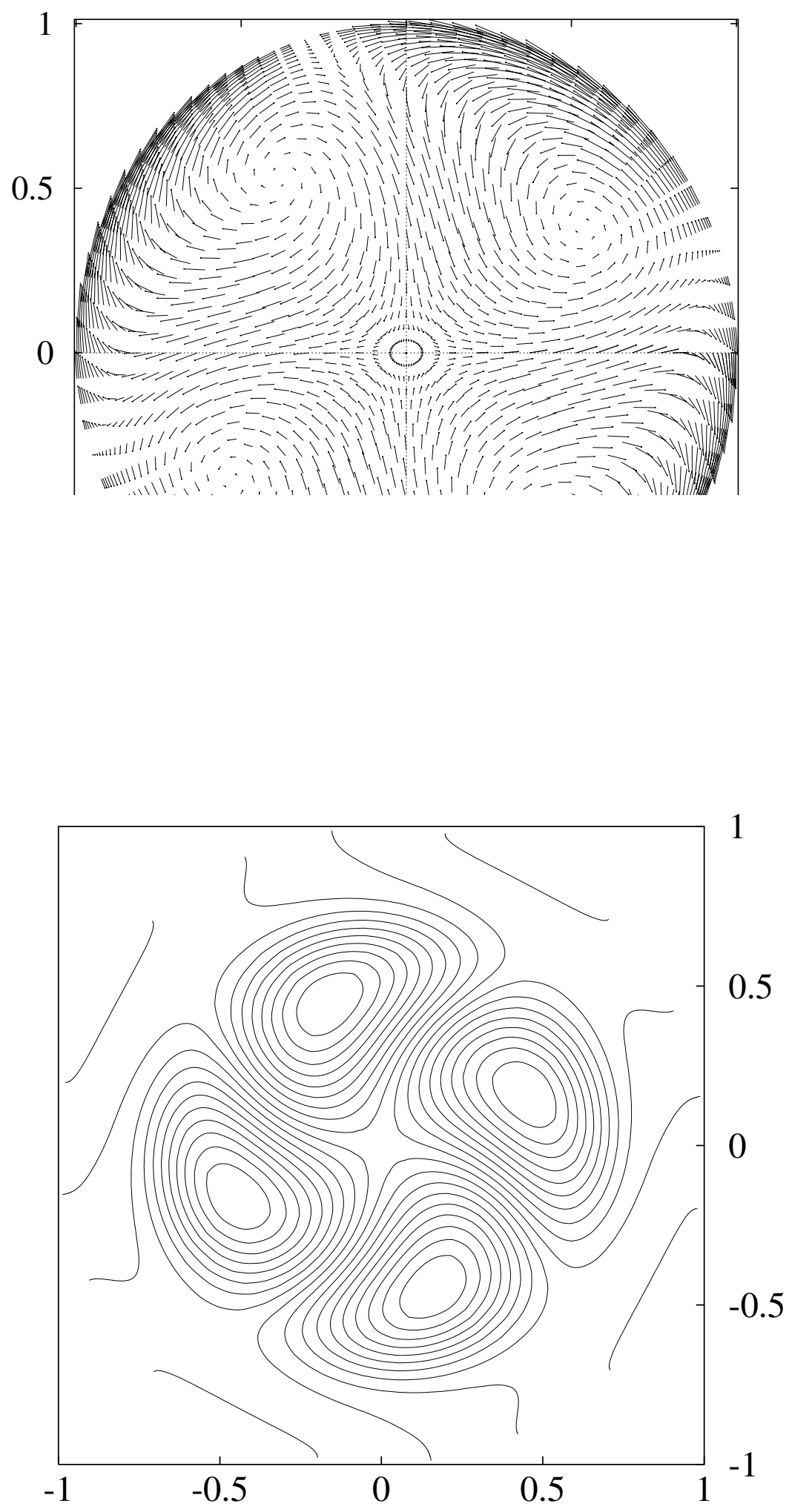

Figure 4: Critical disturbance flow (a) and temperature field (b) in a horizontal cut at $z=0.5$ for $R e_{c}=869, \omega_{c}=22.7, \operatorname{Pr}=7, \Gamma=1, B i=0$ and $G r=0$. 

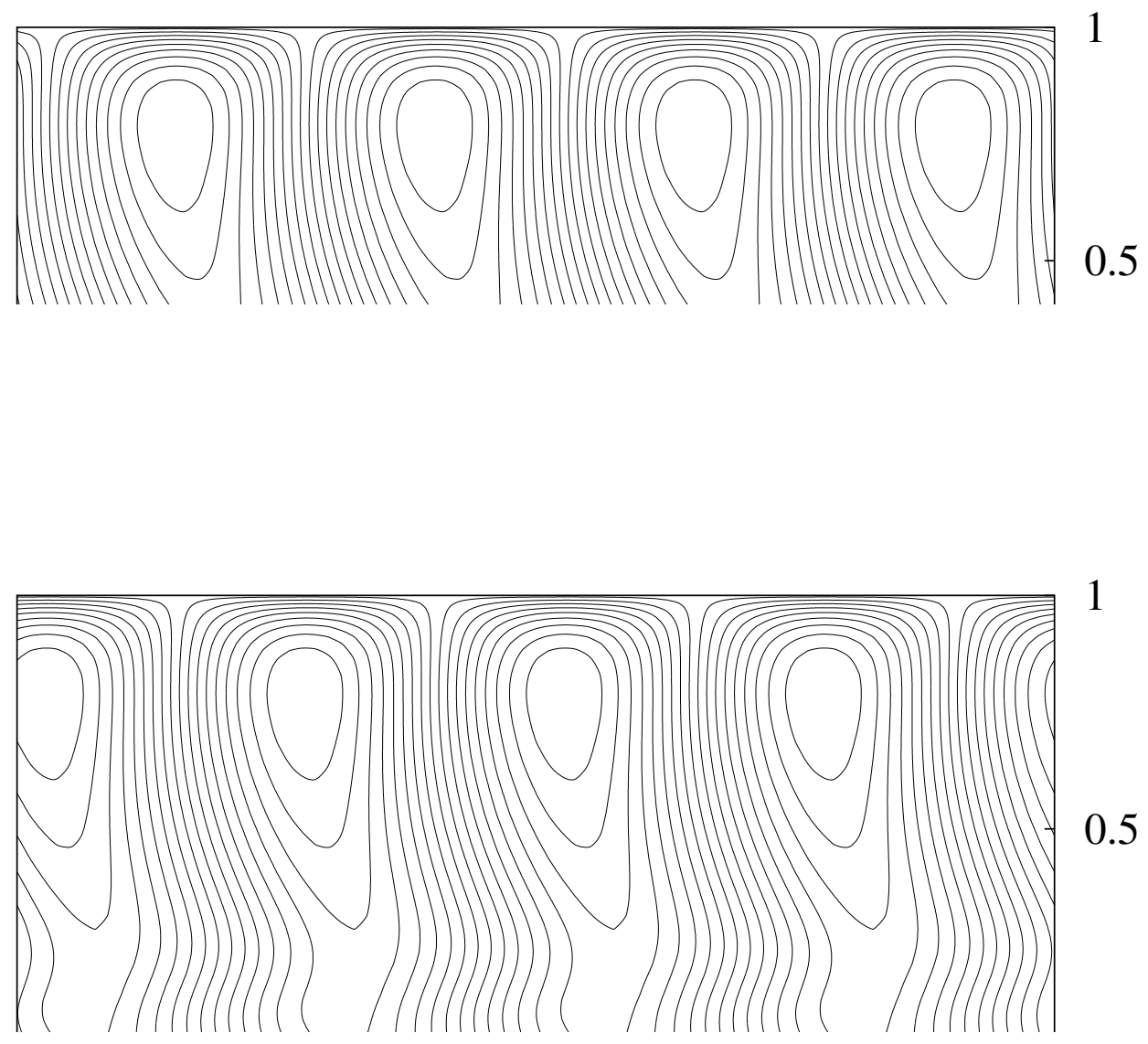


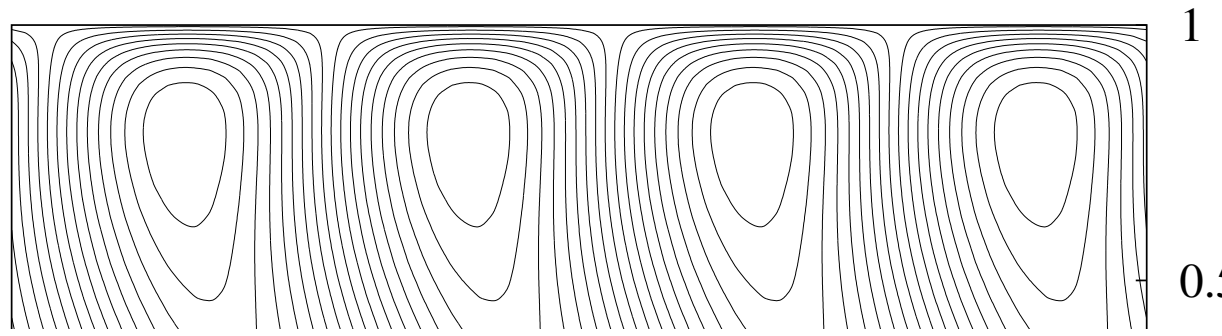

0.5

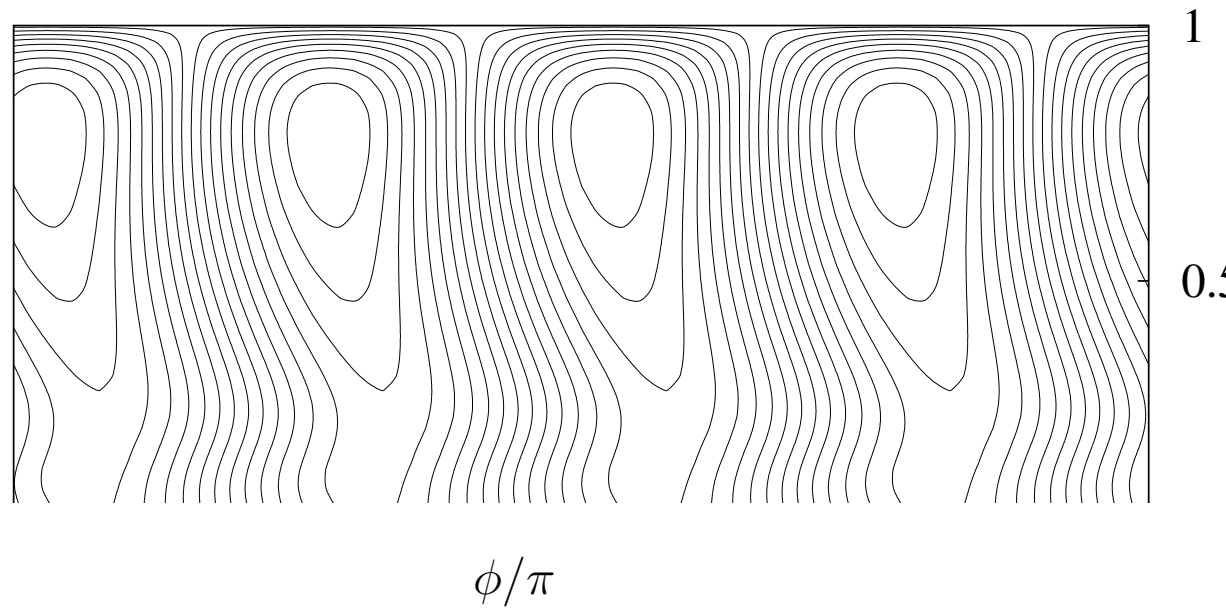

Figure 5: Time-dependence of the critical disturbance temperature field on the free surface (surface hydrothermal wave) for $R e_{c}=869, \omega_{c}=22.7, \operatorname{Pr}=7, \Gamma=1, B i=0$ and $G r=0$. The isotherms are shown at times 0 (a), $1 / 4$ (b), $1 / 2$ (c) and $3 / 4$ (d), in units of $2 \pi / \omega_{c}$. 